\title{
Processo de vigilância em saúde do trabalhador
}

\author{
Worker's Health Surveillance
}

Jorge Mesquita Huet Machado 1

\footnotetext{
1 Coordenação de Saúde do Trabalhador, Diretoria de Recursos Humanos, Fundação Oswaldo Cruz. Av. Brasil, 4365, Prédio do Quinino, salas 209/210 Rio de Janeiro, $R J$ 21045-900, Brasil.
}

\begin{abstract}
This paper is part of a broader discussion on the need for more in-depth study of workers' health surveillance practices, which are most often developed empirically, without welldefined theoretical or technical foundations. The paper presents a concept of surveillance in workers' health as a fulcrum for actions in the relationship between the work process and health. It emphasizes the exposure-based perspective involved in the epidemiological approach. Risk situations and effects are placed in spatial and technological context. The model provides an interdisciplinary approach with a technological, social, and epidemiological basis in a three-dimensional structure. A matrix for planning actions in workers' health surveillance is also presented, focusing on the connections between effects, risks, territory, and activities.
\end{abstract}

Key words Health Surveillance; Worker's Health; Worker's Health Surveillance

Resumo O presente texto insere-se na discussão da necessidade de aprofundamento das práticas de vigilância, muitas vezes, principalmente em países como o nosso, realizadas de forma empírica, carecendo de reflexão sobre suas bases técnicas e teóricas. É apresentado o conceito de vigilância em saúde do trabalhador como articulador das ações de intervenção na relação entre o processo de trabalho e a saúde. A partir do olhar da epidemiologia, são situados riscos e efeitos em um contexto espacial e tecnológico. São descritos cenários recentes, relacionando suas influências e as características formadoras de um modelo de vigilância em saúde do trabalhador, destacando dos aspectos estruturais do modelo um tripé interdisciplinar formado por abordagens sociais, tecnológicas e epidemiológicas. É enfatizada a matriz de priorização e planejamento de ações de vigilância em saúde do trabalhador segundo as conexões entre agravos, riscos, território e atividade.

Palavras-chave Vigilância em Saúde; Saúde do Trabalhador; Vigilância em Saúde do Trabalhador 


\section{Introdução}

A implantação de ações de vigilância em saúde do trabalhador desenvolvidas a partir do setor saúde no país é recente. Esse processo é desencadeado por grupos institucionais localizados em vários pontos do Brasil e resulta em uma gama de experiências distintas, voltadas para esse tema. As diferenças observadas estão relacionadas às potencialidades regionais, que giram em torno da força e qualidade da organização dos trabalhadores quanto às questões de saúde e, em termos institucionais, dependem das políticas regionais e da estrutura organizacional, da capacidade instalada, da qualidade dos profissionais envolvidos e de influências advindas das instituições acadêmicas.

O tema comum a todos, embora persistam entendimentos variados, pode ser generalizado pela definição de vigilância em saúde do trabalhador e corresponde a um processo de articulação de ações de controle sanitário no sentido de promoção, proteção e atenção à saúde dos trabalhadores. Esse tema surge da interseção de dois campos de atuação da saúde pública, a vigilância em saúde e a saúde do trabalhador.

\section{Concepções de vigilância em saúde do trabalhador}

A vigilância em saúde do trabalhador, enquanto campo de atuação, distingue-se da vigilância em saúde em geral e de outras disciplinas no campo das relações entre saúde e trabalho por delimitar como seu objeto específico a investigação e intervenção na relação do processo de trabalho com a saúde. Na prática, entretanto, essa concepção não está ainda incorporada em seu sentido amplo, dados sua fragmentação e o pequeno grau de articulação existente entre os componentes diretamente envolvidos nessa relação (Tambellini,1984), a despeito de tentativas de integração interministerial em nível nacional, da institucionalização de instâncias articuladoras regionais (SESRJ, 1991) e mesmo de ações locais, como as observadas, por exemplo, no Programa de Saúde dos Trabalhadores da Zona Norte de São Paulo (Costa et al., 1989).

O pressuposto da atuação na vigilância em saúde do trabalhador é a articulação entre os controles de exposição e efeito, como indicado pelos pragmáticos gestores do sistema dos EUA, segundo o grupo coordenado pelo Dr. Alexander Langmuir. A vigilância de riscos e a de doenças não podem ser isoladas uma da ou- tra. O sucesso na caracterização de riscos associados com diferentes indivíduos ou ocupações, em conjugação com a informação toxicológica e médica relativa aos riscos, pode sugerir indústrias ou grupos ocupacionais apropriados para a vigilância epidemiológica.

Em contrapartida, um padrão não usual de saúde em certas indústrias ou ocupações é elucidado por uma vigilância de efeitos sobre a saúde e será mais explicado por uma vigilância de agentes causadores potenciais.

Poucas entidades nosológicas são suficientemente específicas em relação a suas causas a ponto de diminuir a necessidade da vigilância de riscos. Alguns agentes têm efeitos específicos, que tornam relativamente óbvia a tarefa de vigilância das doenças. Há, por outro lado, vasto campo em que as exposições são complexas, e os efeitos na saúde são diversos, o que atrela necessariamente a resolução ao esforço combinado de uma vigilância de causa e efeito (Sundin et al., 1977, apud Froines et al., 1989).

Essa concepção articuladora da ligação entre esses pólos de atuação da vigilância é ressaltada, segundo nosso entendimento, pelo conceito de vigilância em saúde e introduz a necessidade de que a vigilância em saúde do trabalhador incorpore como objeto de suas ações as situações de risco e seus determinantes tecnológicos e sociais.

Por sua vez, o conceito de vigilância em saúde apresenta distintas formulações e entendimentos. Pode ser visto de forma restrita, como monitoramento de doenças, resultante da conjugação do atendimento clínico e do acompanhamento de populações expostas por meio de indicadores biológicos de exposição e de efeitos subclínicos, como induz a tradução da expressão inglesa medical surveillance (Health \& Safety Executive, 1990). Esse entendimento, a nosso ver, refere-se exclusivamente à vigilância médica (Matte et al., 1990) ou vigilância da saúde (Salgado \& Fernícula, 1988).

Por outro lado, o conceito de vigilância também está associado às ações sistemáticas de coleta, análise e disseminação de dados, de acordo com a XXI Assembléia Mundial de Saúde de 1968 e, mais genericamente, relacionado às recomendações de articulação com serviços de saúde, programas de saúde e pesquisas epidemiológicas enunciadas pelo conceito de vigilância em saúde pública proposto por Thacker \& Berkelman (1988) e adotado pelos Centros de Controle de Doenças no EUA. Essas concepções podem ser sintetizadas no entendimento de que "vigilância é informação para ação”, conforme apresentado por Wünsch Filho et al. (1992), tendo como referência a vigi- 
lância epidemiológica restrita à coleta, análise e programação de ações de detecção de situações de risco. Essa referência pode servir de ponto de partida para ações de intervenção, o que, teoricamente, se encontra no campo de ação da vigilância; sua prática, porém, por requerer outros métodos e técnicas, não se adequa ao modelo de organização de serviços existentes nos sistemas de saúde. Vigilância é informação para ação, pressupondo que as ações pertençam ao campo da vigilância.

Nesse sentido, o conceito de vigilância em saúde definido por Mendes (1994) situa as ações de saúde no âmbito de um dado território, o distrito sanitário, propondo uma mudança de referência em relação ao modelo assistencial tradicionalmente adotado; a nova perspectiva inclui promoção de saúde, atuação nos determinantes sanitários, coleta, análise e disseminação de informações sanitárias e atenção clínica. De acordo com esse modelo, estabelecer o território como elemento integrador das ações de assistência à saúde dos trabalhadores e de prevenção de agravos relacionados ao trabalho é essencial na concepção de vigilância em saúde do trabalhador aqui apresentada.

Por sua vez, a saúde dos trabalhadores constitui um dos objetos integradores das ações de saúde pública por seu potencial articulador das ações de vigilância sanitária, de vigilância epidemiológica e de serviços de saúde, as três grandes áreas de atuação do setor saúde, segundo Cordoni Junior (1988). A introdução da vigilância em saúde do trabalhador, portanto, representa a possibilidade de estabelecer conexões entre suas respectivas instâncias executoras. Essa rede interna do SUS, estabelecida pelo desenvolvimento sistemático das ações de vigilância em saúde do trabalhador, apresenta como característica decorrente de seu objeto a conexão com instituições além do sistema de saúde em termos restritos, chegando a configurar uma série de pontes intersetoriais.

Deve ser destacado o fato de que equiparar a vigilância em saúde e, em conseqüência, a vigilância em saúde do trabalhador às concepções restritas de vigilância da saúde ou vigilância médica e a de vigilância epidemiológica, mais do que configurar questões semânticas, tem conseqüências importantes na definição de competências institucionais relativas à possibilidade de incorporação de ações de intervenção nos ambientes de trabalho.

\section{Origens e contextualização}

As ações de vigilância em saúde do trabalhador são influenciadas mais diretamente pelas contribuições da medicina social latino-americana e da experiência italiana de reforma sanitária, que enfatizam algumas características e princípios norteadores dessas ações. Cabe ressaltar as seguintes concepções do pensamento da medicina social latino-americana:

1) a determinação social da saúde;

2) a relativização de métodos quantitativos na análise da associação entre causa e efeito;

3) a integração de diferentes disciplinas em torno da discussão da saúde;

4) o desenvolvimento de práticas e gestões participativas em saúde;

5) a compreensão da dinâmica entre sujeito individual e coletivo.

Por sua vez, a reforma sanitária italiana (Berlinguer, 1983) traz em seu bojo os princípios básicos do chamado modelo operário italiano (Odonne, 1986; Laurell \& Noriega, 1989) não delegar, não monetarizar os riscos e buscar a validação consensual em grupos homogêneos -, esses princípios foram interpretados à luz do neopreventivismo do movimento sanitário brasileiro e incorporados nas experiências de desenvolvimento de ações de vigilância em saúde do trabalhador no interior do sistema de saúde na década de 1980, inicialmente com forte cunho sindical e acadêmico, em São Paulo em 1984 (Augusto et al., 1986), seguido por Rio de Janeiro, Minas Gerais e Bahia no final dos anos 80 e, posteriormente, em 1990, generalizado pela lei Orgânica de Saúde, base da reforma sanitária brasileira e do Sistema Único de Saúde - SUS (Dias, 1994).

Esse processo de introdução de ações de vigilância em saúde do trabalhador no SUS carece, no entanto, de consolidação institucional, de reconhecimento social mesmo entre os setores diretamente envolvidos (saúde, trabalho e previdência social), de aprofundamento conceitual e da devida abrangência territorial de atuação. Apesar dessa carência, com a criação dos Programas de Saúde do Trabalhador - PSTs no âmbito de estados e municípios, ocorre a disseminação de ações de vigilância em saúde do trabalhador, em um mimetismo alternativo às ações tradicionais de saúde pública e consoante com os pressupostos do novo modelo assistencial, oriundo da concepção associada à de distrito sanitário. Entretanto, a implantação de ações num sistema em crise financeira e de pessoal técnico - condicionantes devastadores, dadas as características do trabalho em saúde: alta incorporação técnica pelos agentes e tra- 
balho intensivo - é problemática e, muitas vezes, considerada diletantismo e vista com desconfiança pelos gestores regionais, zelosos de não ameaçar empresas e empresários que apóiam politicamente os respectivos governos e preocupados com os problemas oriundos da crise estrutural do setor.

Por outro lado, nos últimos 10 anos, influências da prática cotidiana da vigilância em saúde do trabalhador também podem ser notadas, por contraposição e por interlocução entre instituições, empresas e trabalhadores, como a discussão permanente de limites de tolerância à exposição aos agentes químicos no ambiente de trabalho. Estudos epidemiológicos e toxicológicos e o processo de negociação estabelecido caso a caso (Mattos et al., 1995) muitas vezes se contrapõem aos valores instituídos pela legislação e se incorporam ao debate interno da Higiene Industrial (Castleman \& Ziem, 1988; Arcuri \& Cardoso, 1991) na constante perseguição dos patamares seguros para os valores dos indicadores de exposição biológicos e ambientais, para que cheguem os primeiros à normalidade fisiológica e ao mínimo tecnicamente possível nos índices ambientais.

Outro exemplo de temática recorrente é o estabelecimento de nexo causal entre as condições de trabalho e os casos de doenças, em que a fragmentação institucional tem efeito restritivo, e a previdência social, por intermédio da perícia médica do Instituto Nacional de Seguridade Social-INSS, tem a palavra final. Em locais onde houve uma integração com outros órgãos do subsistema de saúde do trabalhador, como em Belo Horizonte com a criação do Núcleo de Saúde do Trabalhador-NUSAT , ou onde são estabelecidos atendimentos direcionados aos trabalhadores no SUS, com a criação de centros de referência, foi significativamente ampliado o número de doenças relacionadas ao trabalho (Buschinelli, 1994), demonstrando a existência de epidemias submersas na incompetência institucional calcada na deformação técnica, na falta de articulação entre as ações de avaliação clínica e aquelas realizadas no local de trabalho, e nos critérios de avaliação, voltados para a diminuição de pagamento de prêmios do seguro.

Um terceiro tema permanente de embate e conseqüente amadurecimento refere-se à competência institucional para realização de ações relacionadas às condições de trabalho e suas repercussões na saúde; a hegemonia, detida pelas Delegacias Regionais do Trabalho-DRTs, é ameaçada pelo SUS e pela burocratização de suas ações, ainda que observem uma tendência à flexibilização pelo contato interinstitucio- nal e uma contaminação de métodos, de prioridades e de pessoas que atuam em ambos os campos institucionais. A entrada nas empresas para realização de investigações nos locais de trabalho é cercada de entraves e explicações relacionadas ao jogo de legitimação social do SUS como órgão fiscalizador dos locais de trabalho. A atuação nos determinantes sanitários - pressuposto do paradigma da medicina social latino-americana e, por conseguinte, da saúde do trabalhador (Tambellini et al., 1986) ou mesmo nas causas mais imediatas e evidentes sofre resistência mascarada em discussão de competências que são enfrentadas, permanentemente, de maneiras distintas e evolutivas pelos grupos de inspeção.

$\mathrm{O}$ atual modelo brasileiro de vigilância em saúde do trabalhador configura-se nesta polarização: de um lado, a perspectiva de ampliação da atuação institucional, aliada ao movimento sindical relativo às condições de saúde e trabalho, implanta ações de saúde do trabalhador no ainda incipiente SUS; de outro, a atuação institucional, restrita às ações das DRTs, aliada a um controle gerencial interno das empresas estabelece, a partir de pressões e políticas industriais, os parâmetros de autovigilância, constituindo um modelo patronal de intervenção (Gawryszewski, 1988) ou que, simplesmente, se demonstra ineficaz (Oliveira, 1994). Em síntese, esse processo de conflito e de interação está presente no modelo brasileiro de vigilância em saúde do trabalhador.

Esse encontro entre instituições, empresas e trabalhadores apresenta posicionamentos que transformam os pressupostos iniciais oriundos da saúde pública em campo de debate de diferentes paradigmas, sendo o resultado das mudanças observadas a reconstrução de uma área interdisciplinar na sua própria essência devida à interação e ao fortalecimento ou esvaziamento dos argumentos científicos e político-institucionais dos atores envolvidos.

Dessa forma, é constituído um sistema de vigilância em saúde do trabalhador a partir de interações, superposições e conflitos, que conformam um campo de poder, onde forças sociais disputam o controle da burocracia, mediante o direcionamento técnico e administrativo. Entretanto, muitas vezes as forças mais eficazes nessa disputa não estão preparadas para a execução dos pressupostos institucionais, pois as mudanças e os avanços políticos e técnicos esbarram na inércia estrutural do estado e suas instituições, fazendo com que a evolução se dê em ondas e em várias direções. 


\section{Pressupostos metodológicos da vigilância em saúde do trabalhador}

Uma intervenção interdisciplinar em vigilância tem um potencial de superação das restrições técnicas das abordagens disciplinares. Os componentes tecnológicos, epidemiológicos e sociais dessa ação interdisciplinar atuam como mediadores da relação entre processo de trabalho e saúde. Logo, ao levarmos em consideração esses três componentes estruturais (Figura 1) e a dinâmica dessa relação, somos obrigados a pensar e agir interdisciplinarmente.

Componentes sociais, como a situação econômica, organização e consciência dos trabalhadores, fazem parte da análise do processo de trabalho tão fortemente quanto as avaliações de risco e do tipo de tecnologia utilizada em determinado processo de produção, que se configuram como os principais componentes tecnológicos, fundamentais, por sua vez, no entendimento das condições de saúde e dos próprios indicadores que serão utilizados para sua avaliação. Os componentes sociais e tecnológicos são essenciais por contextualizar o componente epidemiológico.

Nessa representação esquemática há uma gama de conteúdos implícitos que se relacionam com as dimensões individuais e coletivas da saúde dos trabalhadores inerentes ao campo delimitado pela epidemiologia, que será abordado adiante.

Diante da constatação consensual de que os corporativismos institucionais e disciplinares dos técnicos envolvidos nas ações criam dificuldades objetivas para o desenvolvimento de atividades, a abordagem interdisciplinar é talvez o pressuposto básico, sem o qual não há condição de entendimento do objeto da vigilância em saúde do trabalhador. A relação entre processo de trabalho e saúde é essencial para a construção de ações de intervenção no

Figura 1

Componentes Estruturais da Abordagem Interdisciplinar da Relação Processo de Trabalho e Saúde.

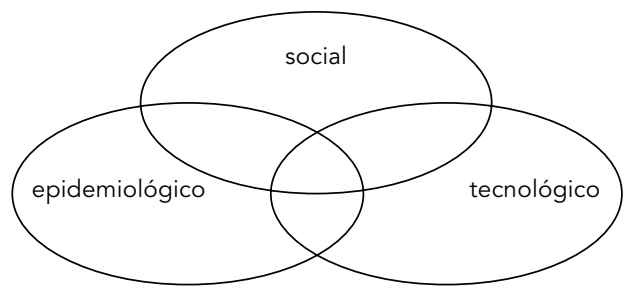

processo de trabalho, bem como para a avaliação dos resultados das intervenções visando à saúde dos trabalhadores.

A atuação de investigação e intervenção pode ocorrer em qualquer ponto ou componente estrutural, desencadeando repercussões em todo o sistema definido pela relação entre processo de trabalho e saúde sob vigilância.

Em termos metodológicos, as ações de vigilância de saúde do trabalhador, além de sistemáticas, estabelecem, em relação a cada caso, um processo específico, construindo a dimensão temporal intrínseca a cada ação de vigilância. Essa é desencadeada em etapas, num processo de aproximação sucessiva (Machado et al., 1992), com aprofundamento e abrangência cada vez maiores, ou seja, grupos de risco são inicialmente delimitados a partir de informações gerenciais e por meio da articulação das ações de intervenção, investigação e dos serviços de atenção à saúde. São realizadas avaliações em que a heterogeneidade dos grupos homogêneos de risco é evidenciada e motiva novas estratificações. Paralelamente, estabelecese um processo de negociação das melhorias das condições de trabalho, e as intervenções propostas nesse âmbito são avaliadas por indicadores sociais, tecnológicos e epidemiológicos. Levam-se em conta as condições tecnológicas da própria empresa, o estabelecimento de prazos e metas para as medidas a serem adotadas. Portanto, embora o período previsto para a intervenção seja virtualmente ilimitado, o estabelecimento de etapas com metas a serem cumpridas confere racionalidade e visibilidade ao processo de vigilância. Em síntese, o método de vigilância em saúde do trabalhador se aproxima dos desenhos de estudos epidemiológicos definidos por Kauppinen (1994) para os estudos da exposição industrial, com nível de formalização do desenho do estudo menor e mais flexível, mas, entretanto, com relação interdisciplinar mais intrínseca.

São incorporados parâmetros técnicos de priorização das ações de acordo com análises que integrem a observação de situações críticas segundo critérios de gravidade e magnitude, por região, por setores de atividade econômica, por agravos à saúde (efeito) e por risco (tipo de exposição). Essa matriz científica é a base de interlocução com as demandas sociais do movimento sindical e de outras instâncias institucionais, os poderes executivo, legislativo e judiciário.

O processo de estabelecer o risco é apresentado de forma sintética, linear e esclarecedora por Hernberg (1994), no editorial comemorativo de 20 anos da revista Scandinavian 
Journal of Work and Environmental Health, dividindo-o em etapas e em duas fases, uma essencialmente científica, e outra gerencial. A primeira fase constitui:

a) designar o que é perigoso e quais são os possíveis danos decorrentes;

b) estabelecer como são causados os danos e esclarecidas as situações ou os fatores de agravamento e de proteção, ou seja, se os danos podem ou não ser menos ou mais graves na presença de outros fatores;

c) determinar a severidade do risco. Estudos quantitativos comparando diferentes níveis de exposição devem ser realizados a fim de permitir a conclusão quanto à quantidade de exposição que causa cada tipo de dano e se é possível determinar um limite seguro de exposição;

d) realizar extrapolações no campo da saúde pública e, mais especificamente, no campo da saúde ocupacional a fim de tornar clara a magnitude do problema, ou seja, a quantidade de casos de doença diante de uma dada situação de exposição.

Nesse ponto, acaba o domínio científico, mas não a contribuição científica, e a função administrativa de gerenciamento de riscos se inicia, configurando uma segunda fase, a de vigilância das situações de risco e de seus efeitos previamente definidos. Essas duas fases delimitam o que é investigação científica e o que é vigilância; aqui nos deteremos na vigilância e nos seus aspectos científicos. A fase de vigilância é essencialmente contínua, tendo por base um direcionamento social estabelecido; no caso da vigilância em saúde dos trabalhadores, um relacionamento estreito com os trabalhadores, um sistema de informações e um aparato de intervenção institucional são elementos estruturais de um sistema de vigilância. As situações críticas tornam-se objeto de vigilância a partir de denúncias, de movimentos sociais e de diagnósticos técnicos específicos ou por analogia a condições similares em que foram estabelecidas situações de alto risco.

\section{Contextualização epidemiológica}

O componente epidemiológico essencial na estrutura interdisciplinar da saúde do trabalhador, conforme apresentado na Figura 1, será destacado por seu aspecto articulador.

A forma de intervenção da saúde pública implica a valorização do método epidemiológico na execução das ações em VST, sendo elemento de sua validação. A epidemiologia, portanto, se constitui em ferramenta de análise e aglutinação de distintas abordagens ao relacio- nar situações sanitárias, sejam no nível molecular, individual ou de grupos com condições ambientais e históricas, permitindo a construção de redes interdisciplinares e configurando um contexto epidemiológico de interpretação e avaliação.

Em termos operacionais, a epidemiologia organiza e relaciona a informação de saúde com o processo de trabalho; identifica, descreve e analisa características comuns e heterogêneas das repercussões sanitárias em distintos estratos de trabalhadores; e possibilita a construção de grupos estratificados segundo características comuns relacionadas ao processo de trabalho. Nesse sentido, a análise sucessiva da heterogeneidade dos grupos homogêneos é ferramenta fundamental.

Estudos da contaminação por agentes químicos têm demonstrado de maneira enfática que o método epidemiológico, embora essencialmente quantitativo, carece de uma relação interdisciplinar que incorpore enfoques qualitativos, que, por sua vez, serão decisivos na interpretação das situações descritas ou analisadas epidemiologicamente e na elaboração de estratos e recortes a serem comparados (CONSEST, 1993; Machado et al., 1992).

As discussões de modelagem e de efeitos confundidores revestem-se, no caso do uso da epidemiologia nos processos de vigilância, de dois aspectos centrais:

1) a epidemiologia, como essencial para a integração interdisciplinar, ao constituir-se em um instrumental de aglutinação de diversas abordagens de identificação das características do processo de trabalho e do processo saúde/doença, transformando-as em variáveis de exposição e efeito;

2) a identificação de efeitos confundidores ao incorporar o processo de trabalho como categoria fundamental nas ações de vigilância, pois há relação direta entre a profundidade do entendimento do processo de trabalho e a possibilidade de quantificação da exposição e da especificidade dos estratos recortados nas análises epidemiológicas.

Esses dois aspectos integram-se e formam o núcleo da investigação em saúde do trabalhador relacionado à identificação de situações de risco e à avaliação de impactos sobre a saúde provocados por procedimentos de gerenciamento de riscos ou mudanças tecnológicas. Dessa forma, constrói-se o agir epidemiológico em saúde do trabalhador, o que possibilita, pela clareza e caráter incisivo de seus resultados, estabelecer diálogo interno, no setor saúde, e externo, principalmente com setores ligados à política industrial, do trabalho e do meio ambiente. 
No campo acadêmico e institucional, a epidemiologia tem sido usada para situar os eventos sanitários em contextos espaciais, temporais e em grupos de indivíduos, por meio de estudos com diferentes magnitude em relação à abrangência da observação, articulando análises mais amplas com outras, pontuais. No caso da saúde do trabalhador, os estudos por setor ou ramo produtivo têm sido promissores. $\mathrm{O}$ uso da epidemiologia nesses casos situa os problemas de saúde em contextos espaciais, mapeando a morbimortalidade; identifica tendências temporais e grupos de trabalhadores com maiores riscos, mediante estudos com distintas dimensões, em que abordagens de maior magnitude e menor profundidade se complementam com recortes mais profundos e de menor abrangência. Esse mapeamento em diferentes níveis de complexidade, juntamente com uma abordagem interdisciplinar dos componentes sociais, tecnológicos e epidemiológicos recortada por atividades econômicas, vemse constituindo em uma metodologia característica das investigações recentes em saúde do trabalhador (Facchini, 1986; Laurell \& Noriega, 1989; Minayo, 1992; Machado, 1995).

A simplificação essencial que o método epidemiológico opera nesse processo diz respeito à conjugação das abordagens disciplinares da medicina do trabalho, da higiene industrial e da engenharia de segurança em uma abordagem clássica de saúde ocupacional. Pode também articular-se de forma interdisciplinar ao incorporar o processo de trabalho como categoria e objeto fundamental de sua reflexão e intervenção, configurando-se em abordagem de saúde do trabalhador.

Portanto, os dados de histórias ocupacionais e de avaliações clínicas, os indicadores biológicos de doses internas e de efeitos, as avaliações ambientais, as identificações de situações de risco potencial, as análises ergonômicas e de outras disciplinas, as avaliações qualitativas e de percepção de riscos com os próprios trabalhadores e técnicos são dimensionadas por intermédio da epidemiologia nesse processo de simplificação, estabelecendo diferenças e similaridades dos efeitos ou impactos na saúde às também similares ou distintas exposições.

Deve ser ressaltada a necessidade de aperfeiçoamento das abordagens qualitativas na definição da exposição, pois, no debate interno da epidemiologia, só tem sido aprofundado o importante desenvolvimento de técnicas de análise quantitativas e estatísticas, como no caso das matrizes de exposição (Bouyer \& Hémon, 1993).
Porque a vigilância atua em situações onde a investigação científica já estabeleceu os riscos, verifica-se uma relativização da limitação de que os estudos epidemiológicos sejam conclusivos a posteriori. A abordagem por setor ou ramo econômico, ou mesmo por tipo de atividade de trabalho permite, com a generalização de uma intervenção no processo de trabalho de um dado ramo ou setor, gerar um impacto preventivo à distância, ampliando desse modo o impacto sanitário das ações em saúde do trabalhador, pela superação do campo restrito do processo de investigação. Essa superação estabelece a perspectiva da construção de uma epidemiologia da exposição não só no sentido de sua qualificação, mas também de intervenção e avaliação, percorrendo o sentido inverso do raciocínio da causalidade.

Por sua vez, o aprofundamento do estudo da exposição abre uma alternativa de entendimento do processo de trabalho enquanto um determinante da situação de saúde, ou seja, abordagens mais sofisticadas de quantificação da exposição tendem a esclarecer situações de risco e suas variações, permitindo a visualização de uma epidemiologia da exposição (Kauppineen, 1994).

\section{Matriz da vigilância em saúde do trabalhador}

Será apresentada a seguir uma síntese reflexiva das distintas formas de delimitação dos objetos e de suas inter-relações nos processos de vigilância em saúde do trabalhador por tipo de efeito, agravos; por tipo de exposição, riscos ou atividades; e segundo delimitação espacial.

\section{Agravos}

A maior parte das ações de saúde do trabalhador nos sistemas de saúde foi historicamente constituída a partir da criação de um atendimento capaz de evidenciar casos relacionados ao trabalho no sistema de saúde (Baker, 1989). Essa estratégia de vigilância é considerada auxiliar, segundo o Ministério do Trabalho dos EUA, e deveria estar integrada a um sistema de vigilância.

Por essa via, as ações de vigilância em saúde do trabalhador surgem a partir de procedimentos de vigilância médica e, por sua vez, induzem modificações nos ambientes de trabalho.

Em termos gerais, a identificação e a avaliação da magnitude e da transcendência dos agravos à saúde relacionados ao trabalho per- 
mitem a priorização de questões relevantes segundo uma perspectiva epidemiológica. Essa abordagem depende da estrutura de atendimento e da capacidade diagnóstica específica do sistema de saúde e, por conseqüência, da política institucional do próprio SUS.

A busca ativa de casos e a criação de programas de vigilância permitem a identificação de determinantes do agravo, por meio da análise da história ocupacional, e a delimitação de novas estratégias de controle de riscos.

Algumas experiências nacionais de investigação de agravos relacionados ao trabalho se transformaram em teses e análises acadêmicas (Machado et al., 1989; Echternacth, 1991; Wünsch Filho, 1992; Augusto et al., 1993; Castro, 1994; Ferreira, 1994; Rocha et al., 1994; Setimi, 1995). Vários desses textos foram viabilizados por ações de vigilância e se tornaram elementos dinamizadores de práticas institucionais, em clara interdepêndencia de processos de vigilância e de geração de conhecimentos.

Os agravos destacados como os mais comumente encontrados na literatura dos estudos epidemiológicos relacionados às condições de trabalho são os cânceres, as doenças cardiovasculares e neurológicas, as desordens musculoesqueléticas, as conseqüências reprodutivas e, recentemente, os efeitos dos campos eletromagnéticos (Axelson, 1994), além das doenças pulmonares e dermatológicas, e dos casos de hipoacusias, que compõem a tríade de maior prevalência dos registros internacionais no período anterior à pandemia de lesões por esforços repetitivos, LER, hoje observada.

Por sua vez, a revista Occupational Medicine State of the Art Reviews, em 1990, publicou vários artigos de revisão atualizada de temas, destacando a vigilância médica do câncer e seu monitoramento biológico (Cone \& Rosenberg, 1990), das doenças pulmonares (Balmes, 1990), das doenças renais (Kosnett, 1990), das doenças neurológicas (Cone et al., 1990) e das hepatotoxinas relacionadas aos ambientes de trabalho (Harrison, 1990), e estabelecendo diretrizes de controle de agravos relacionados à exposição principalmente a agentes químicos e a atividades de risco comprovado ou de forte suspeita.

Essas indicações são relativamente restritas a efeitos específicos, deixando de lado o efeito difuso das condições de trabalho na saúde. Deve-se, portanto, ressaltar que os casos sem características clínicas específicas podem passar despercebidos pelas avaliações de saúde e, por isso mesmo, dependem mais ainda da abrangência e qualidade dos atendimentos de refe- rência e do grau de articulação do sistema de vigilância em saúde do trabalhador. Essa estratégia, como, aliás, as demais, deve estar ligada a outras abordagens, evitando que a intervenção seja restrita ao nível individual e clínico.

Por outro lado, a evidência do agravo e sua relação com as condições de trabalho são pontos críticos em relação ao estabelecimento de mecanismos de resistência por parte das empresas, sendo, paradoxalmente, de grande capacidade legitimizadora de intervenções abrangentes por parte dos agentes sanitários, que poderão influenciar a busca de alternativas tecnológicas de potenciais patogênicos relativamente menores.

\section{$\underline{\text { Riscos }}$}

A abordagem de riscos à saúde do trabalhador permite o controle de causas de acidentes, sejam agentes físicos, químicos e biológicos causadores de agravos, esforços físicos e sobrecargas mentais. Essa intervenção se efetivará basicamente no campo tecnológico, mas terá conseqüências sobre a saúde, que devem ser acompanhadas por meio de indicadores sociais e sanitários. Essa abordagem depende, por outro lado, de prévia concepção sobre os processos determinantes de agravos à saúde. A priorização de um determinado risco surge como resposta a pressões sociais organizadas em conjunto com articulações no campo técnicocientífico e terá maior viabilidade nos casos em que haja relação direta entre agente de risco e danos potenciais à saúde. A identificação consensual de um modelo de determinação do agravo permite a concepção de estratégias de intervenção e, até mesmo, a legitima.

Portanto, o conceito de risco adotado é o de risco potencial, situação ou fator de risco delimitado como um determinante de efeitos adversos, configurando-se em um determinado tipo de exposição a uma situação indesejável.

A metodologia de controle ou eliminação de riscos à saúde do trabalhador mediante diferentes níveis de acompanhamento foi proposta pelo PST-RJ com o objetivo de avaliar riscos relativos à utilização do mercúrio numa indústria de cloro-soda, (Melo \& Barcellos, 1993). Essa abordagem prevê a identificação de indicadores que pemitam o acompanhamento permanente de riscos em todas as etapas compreendidas entre a utilização de substâncias tóxicas e o agravo potencial à saúde. Os passos intermediários compreendem a emissão da substância para o ambiente, a exposição dos trabalhadores em decorrência da contaminação do ambiente de trabalho, a dose individual 
incorporada em conseqüência dessa exposição e os danos estabelecidos clinicamente. Essa abordagem é facilitada nos casos em que predomine um agente de risco de fácil identificação laboratorial e com quadro clínico característico e precoce. Por outro lado, essa estratégia contém a desvantagem de reduzir o contexto de riscos a uma cadeia de eventos pré-estabelecidos. As possíveis interações com outros riscos e os contornos sociais da exposição só poderão ser estabelecidas se ela estiver articulada a outras formas de abordagem da relação entre processo de trabalho e saúde.

A intervenção nos distintos momentos e dimensões das situações de risco requer abordagens distintas, como observa Porto (1994), estabelecendo como níveis de prevenção o indivíduo, o posto/setor, a fábrica e o ambiente.

Como vimos em relação ao conceito de vigilância em saúde do trabalhador, vigilância médica e vigilância da saúde, a substituição do conceito de risco por carga pode resultar em diferenças significativas de entendimento da relação entre trabalho e saúde (Facchini, 1994).

Do ponto de vista quantitativo, o conceito de carga pode homogeneizar os riscos e permitir o somatório de distintos fatores de risco, operacionalizando quantitativamente a exposição potencialmente patogênica decorrente de uma dada atividade laboral. Isso não difere da abordagem epidemiológica, que, mediante análises multivariadas, também realiza essa possibilidade de agregar e comparar situações de desgaste, com a vantagem de ser possível o estabelecimento do impacto de cada componente de risco. A tomada de posição nesse caso, a favor ou contra um ou outro conceito, oculta as verdadeiras dificuldades de manuseio dos resultados de avaliações epidemiológicas. O essencial é a integração das abordagens quantitativas e qualitativas, intrínsecas umas às outras; nesse caso, não é o nome que importa e sim a descrição e a delimitação ou agregação da exposição, que são feitas qualitativamente. A exposição torna-se um objeto e um mesmo objeto, não importando se denominada carga ou risco. Porém, na maioria dos processos de vigilância, a articulação de análises quantitativas e qualitativas é limitada, mesmo em acompanhamentos sistemáticos, dadas as características da formação profissional dos componentes das equipes de vigilância.

Por sua vez, os defensores do conceito de carga estabelecem que o risco é contextualizado qualitativamente e transformado em carga (Brito \& Porto, 1991), estando sua vantagem exatamente no nível das abordagens qualitativas, ou seja, da melhor caracterização da situa- ção de trabalho, e sendo, assim, mais uma forma de entendimento da dinâmica dos fatores de risco, portanto um conceito de ligação entre os riscos e o processo de trabalho, e entre este e os efeitos sobre a saúde.

Por outro lado, devemos levar em consideração que as mudanças permanentes no processo de trabalho e na vida dos trabalhadores constituem elementos de complexidade maior do que aquela que os modelos de análise e categorização de matrizes podem dar conta.

Daí a importância do estudo de casos por meio de abordagens quantitativas e qualitativas específicas, dadas a dimensão e a dinâmica dos fenômenos estudados em ações de vigilância, em que riscos e cargas estão contextualizados em situações específicas de trabalho.

\section{Território}

A abordagem territorial está em consonância direta com os pressupostos do modelo de intervenção por distritos sanitários, em que os determinantes sanitários e os agravos devem ser mapeados e analisados em relação ao potencial impacto sobre a saúde. No caso da vigilância em saúde do trabalhador de um determinado território, a tarefa essencial a ser desempenhada é a localização espacial dos agravos relacionados ao trabalho e às atividades laborais. A identificação das situações de risco ocorre por meio da associação espacial entre exposição e efeito. A limitação dessa via de atuação é a necessidade prévia de uma concepção de risco e de agravos, ou seja, previsão do que deverá ser mapeado, o que resulta na tendência de homogeneização de resultados e na superficialidade em relação ao entendimento de processos específicos e suas dinâmicas, podendo contraditoriamente, constituir-se em uma forma de difusão de ações e, portanto, de identificação de novas situações críticas.

A adoção de uma abordagem territorial está relacionada com a implantação e a integração do modelo assistencial previsto para o SUS e com questões objetivas de operação de atividades de vigilância, como o rastreamento de atividades informais de trabalho realizadas no espaço das ruas e, mesmo, clandestinamente.

Devem coexistir mapeamentos de riscos e agravos específicos em níveis micro e macrorregionais e até por empresa, setor da empresa e mesmo de postos de trabalho e por atividades. A especificidade dessa forma de abordagem é a possibilidade de nos aproximarmos da totalidade dos determinantes e dos efeitos, ou seja, uma abordagem só limitada espacialmente e pelos métodos e critérios do mapeamento. 
É também uma estratégia local de superação do poder imperial de grandes empresas em relação ao poder público local, que as contextualizariam em uma ação geral, tendo por base a racionalidade regional.

O território age ainda como fator determinante em si e não só como espaço aglutinador de situações de risco e efeitos, pois nas ações de vigilância estão presentes também os condicionantes espaciais institucionais, os de organização dos trabalhadores e os geográficos de várias qualidades, como o local de trabalho, os deslocamentos e a própria situação geofísica.

\section{Ramo de atividade econômica}

A base de atuação da vigilância por ramo de atividade econômica segue a lógica da organização dos trabalhadores, o que viabiliza a participação sindical na priorização, delimitação e acompanhamento das ações de vigilância. Por outro lado, um dado ramo de atividade guarda internamente similaridade no processo de trabalho, o que facilita a discussão e o entendimento dos determinantes de saúde. Diante da evidência de determinado tipo de agravo (acidentes ou casos clínicos relacionados a uma tecnologia) ou sua forma de aplicação, ou mesmo a própria cultura do setor em relação à saúde e segurança no trabalho, pode-se estabelecer um processo de intervenção e negociação de controle e mudanças no processo de trabalho, seja em sua base tecnológica ou de organização de trabalho, o que virtualmente poderá eliminar o risco de acidentes e de adoecimento relacionados ao trabalho.

"O caminho que se procura trilhar para superar as fronteiras do técnico tem sido buscar trabalhar em conjunto com os trabalhadores organizados através de seus sindicatos. Desta forma deixa de ser absolutamente necessária a intervenção sobre o ambiente de todas as empresas de um determinado ramo de atividade. Uma intervenção articulada com a organização sindical dos trabalhadores em indústrias de calçados e artefatos de couro, por exemplo, permite que a análise de uma única empresa do setor tenha repercussão ampla nesta categoria de trabalhadores e as conclusões tiradas podem servir de base para o planejamento de intervenções em outras empresas do ramo e também proporcionar elementos específicos de saúde para negociação de dissídios coletivos” (Wünsch Filho et al., 1992:8-9).

Essas afirmações enfatizam que a característica mais marcante dessa concepção é o seu potencial de intervenção no sentido de a eficá- cia da prevenção tender ao máximo. Todas as outras formas de abordagens caminhariam para esse tipo de intervenção que, no seu limite, tornaria o trabalho totalmente humanizado no sentido de facilitar a relação entre as pessoas.

Entretanto, no processo da negociação, quando são enfrentados interesses de grupos empresariais, há potencialização de limitação de ganhos e velocidade de mudança aquém da esperada, o que propicia retrocessos, nos quais os representantes dos trabalhadores e de instituições públicas tendem a não ter fôlego para o seu acompanhamento, dado o caráter geracional a que está condenada a maioria dos avanços tecnológicos.

Uma conseqüência desse tipo de abordagem é a identificação de atividades de alto risco no interior dos ramos econômicos, como a manutenção das portas das coquerias na indústria siderúrgica, o jateamento de areia seca na indústria naval, a operação de células de $\mathrm{Hg}$ na indústria de cloro-soda, que passam a constituir situações para as quais se buscam alternativas tecnológicas, visando à eliminação de riscos e a sua substituição. Na verdade, embora contextualizadas pelas atividades econômicas, as atividades de risco são os verdadeiros objetos desse tipo de intervenção.

\section{Os pontos cruzados}

Os exemplos concretos, como o referido anteriormente, articulam os pontos da matriz, configurando um modelo relacionado entre eles, como demonstrado na Figura 2.

Alguns exemplos demonstram claramente que a relação entre os pontos da matriz estabelecem e delimitam os objetos de vigilância.

A vigilância da silicose no setor naval delimita as atividades de jateamento para tratamento abrasivo de chapas enquanto situação de risco. Em seu processo de investigação e intervenção é evidenciada a diversidade intrín-

Figura 2

Matriz de atuação em vigilância em saúde do trabalhador.

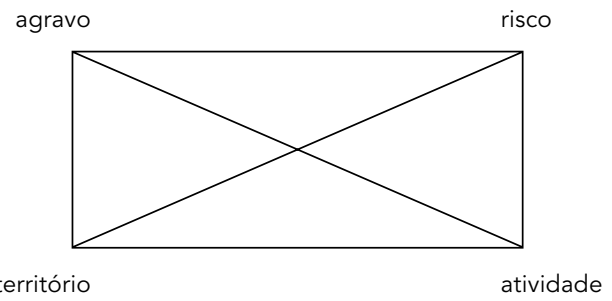


seca às atividades. Verifica-se que, no reparo, a exposição é em geral mais intensa, e seu controle, mais problemático do que nas atividades de construção. A existência de distintas operações que utilizam o tratamento abrasivo em locais que determinam condições ambientais de dispersão de poeira e situações de risco diferenciadas entre si - como os jateamentos de costado, em cabines, em porões e em fundos duplos, e em diferentes tipos de peças, grandes blocos, estruturas fixas, pequenas peças e chapas lisas - estabelece amplo gradiente de exposição e a subdivisão da atividade de acordo com as situações de risco específicas (Machado, 1995). Mesmo a silicose decorrente do jateamento de areia apresenta diferenças, no tempo de latência e no prognóstico, em relação à silicose dos mineiros, dos ceramistas e dos cavouqueiros de poços do Ceará (Mendes, 1986; Echternacht, 1991; CTEPO, 1995; Holanda et al., 1995). Essas situações específicas estabelecem-se pelas características tecnológicas e pelas formas de organização do trabalho, que geram exposições múltiplas e de intensidades variáveis e que empregam contingentes distintos de trabalhadores, estabelecendo resistência biológica e quantidade de exposição distintas durante um dado período de tempo.

Uma atividade pode estar associada a vários tipos de danos, o que é demonstrável na atividade de jateamento, em que está presente um conjunto de situações de risco - poeira, ruído, altura, posições viciosas - que persiste mesmo com a substituição dos abrasivos, devendo, portanto, ser considerados pelas medidas de controle (Machado, 1995).

O caso das lesões por esforços repetitivos é também um exemplo em que distintas atividades, como de digitação, de telefonia, de empacotamento, entre outras (Setimi, 1995), podem levar ao mesmo tipo de dano; quanto maior a gama de atividades causadoras de um tipo de agravo, maior a tendência de aumento de sua incidência e da necessidade de disseminação de estratégias múltiplas de controle de exposição.

Nos casos de um tipo de agravo resultante de várias atividades e de atividade causadora de danos múltiplos é patente a necessidade da territorialização dos eventos, pois a delimitação espacial racionaliza a operação de vigilância, delimitando territorialmente sua intervenção nos efeitos ou nas situações de risco.

Então, tanto quanto um agravo pode ser causado por várias atividades, uma atividade pode causar vários tipos de agravos. Essa multiplicidade é mediada pela exposição, dado que várias atividades podem ter o mesmo tipo de exposição e uma atividade pode conter vários tipos de exposição, o território se constituindo no espaço de intervenção e configurando um quadrilátero de atuação, com dois pólos de intervenção, risco (exposição) e agravo (efeito), e dois pólos de delimitação, atividade (contexto) e território (organização).

\section{Conclusão}

1) o conceito de vigilância em saúde do trabalhador dimensiona a abrangência de sua intervenção na articulação de ações voltadas para as dimensões individual e coletiva no campo da saúde pública em torno da relação entre processo de trabalho e saúde.

A delimitação entre ciência e vigilância é dada pela distinção entre seus objetivos; enquanto a ciência busca a verdade do saber, a vigilância busca o saber para agir. Entretanto, nessa ação compulsiva da vigilância para intervir, é também gerado conhecimento, suprindoa de novos saberes, em ação dialética dos processos de vigilância e de construção da ciência. Em princípio todo processo de vigilância se configura em um modelo de estudo de intervenção, constituindo-se parte do processo de formulação científica, nutrindo-se e nutrindoo de saberes específicos.

Uma delimitação cuja explicitação se faz necessária é a da diferença entre saúde do trabalhador e vigilância em saúde do trabalhador; embora as ações de vigilância estejam inseridas teoricamente no campo da saúde do trabalhador, são as ações em saúde do trabalhador que se inserem na vigilância em saúde do trabalhador, que é um campo de prática articuladora de ações.

Portanto, vigilância é ação em torno de um objeto, no caso da vigilância em saúde do trabalhador, em torno de objetos que alteram, no sentido positivo, a relação entre processo de trabalho e saúde. A informação é elemento deflagrador e de avaliação das ações, sendo fundamento essencial à vigilância.

2) as ações de vigilância em saúde do trabalhador, embora sejam teoricamente interdisciplinares e interinstitucionais, podem, na prática, constituir-se em espaços de lutas corporativas, limitadas a avaliações pontuais e de baixa eficácia na implantação de mudanças nas condições de trabalho. Esse conflito é superado nos processos em que as lutas sindicais e a gravidade sanitária das situações suplantam os muros das empresas. É o caso do benzenismo, da LER, e do hidragirismo, em que se envolveram numerosos atores em diferentes momentos. A delimitação de atividades de alto risco e 
a conseqüente discussão de regulamentações específicas no sentido da eliminação de riscos e substituição tecnológica parecem ser uma tendência positiva e presente nos processos de negociação decorrentes das ações de vigilância.

3) a relação das investigações com processos de tomada de decisão no nível das empresas e do poder executivo é dependente da forma e da força com que se estabelecem interligações entre a academia, os órgãos de vigilância, as empresas e representações dos trabalhadores, bem como das relações entre os poderes executivo, judiciário e legislativo, conformando-se idealmente em um modelo flexível de atuação reflexiva em contato permanente com a realidade e essencialmente participativo, multiprofissional, interinstitucional e interdisciplinar.

\section{Referências}

ARCURI, A. S. A. \& CARDOSO, L. M. N., 1991. Limites de tolerância? Revista Brasileira de Saúde Ocupacional, 19:99-105.

AUGUSTO, L. G. S.; BICHIR, A; FRIETAS, C. U.; LACAZ, F. A. C. \& ESTHER, L., 1986. Vigilância epidemiológica de doenças ocupacionais. Revista Brasileira de Saúde Ocupacional, 54:32-64.

AUGUSTO, L. G. S.; VIGORITO, A. C. \& SOUZA, C. A., 1993. Alterações hematológicas da medula óssea secundárias à exposição do benzeno. Revista Brasileira de Saúde Ocupacional,78:85-92.

AXELSON, O., 1994. Some recent developments in occupational epidemiology. Scandinavian Journal of Work Environment and Health, 20 (special issue):9-18.

BAKER, E. L., 1989. Sentinel Event Notification System for Occupational Risks (SENSOR): the concept. American Journal of Public Health, 79 (suppl.): $18-20$

BALMES, J. R., 1990. Medical surveillance for pulmonary endpoints. Occupational Medicine: State of Art Reviews, 5:499-513.

BERLINGUER, G., 1983. A Saúde nas Fábricas. São Paulo: Ed. Hucitec.

BOUYER, J. \& HÉMON, D., 1993. Comparison of three methods of estimating odds ratios from job exposure matrix in occupational case-control studies. American Journal of Epidemiology, 137:472-481.

BRITO, J. C. \& PORTO, M. F. S., 1991. Processo de Trabalho, Riscos e Cargas à Saúde. Rio de Janeiro: Centro de Estudos da Saúde do Trabalhador e Ecologia Humana, Escola Nacional de Saúde Pública. (mimeo.).

BUSCHINELLI, J. T. P., 1994. Epidemiologia das Doenças do Trabalho no Brasil. Dissertação de Mestrado, São Paulo: Faculdade de Saúde Pública, Universidade Estadual de São Paulo.

CASTLEMAN, B. I. \& ZIEM, G. E., 1988. Corporate Influence on Threshold Limits Values. American Journal of Industrial Medicine, 13:531-559.

CASTRO, H., 1994. Estudo Imunológico de Trabalhadores Expostos à Sílica. Dissertação de Mestrado, Rio de Janeiro: Faculdade de Medicina, Universidade Federal do Rio de Janeiro.
CONE, J. E.; BOWLER, R. \& SO, Y., 1990. Medical surveillance for neurologic endpoints. Occupational Medicine: State of Art Reviews, 5:547-562.

CONE, J. E. \& ROSENBERG, J., 1990. Medical surveillance and biomonitoring for occupational cancer endpoints. Occupational Medicine: State of Art Reviews, 5:563-581.

CONSEST (Conselho Estadual de Saúde do Trabalhador), 1993. Avaliação da Contaminação por Metais Pesados em Trabalhadores de Reparo Naval. Rio de Janeiro: Câmara Técnica da Saúde do Trabalhador na Indústria Naval, S.E.S.-R.J., Informe 1.

CTEPO (Comissão Técnica Estadual de Pneumopatias Ocupacionais), 1995. A silicose na indústria naval do Estado do Rio de Janeiro - Análise parcial. Revista Brasileira de Pneumologia, 21:13-16.

CORDONI JÚNIOR, L., 1988. Sobre a organização do nível central dos seviços públicos de saúde. Saúde em Debate, 22:38-44.

COSTA, D. F.; CARMO, J. C.; SANTOS, U. P.; SETTIMI, M. M., 1989. Programa de Saúde dos Trabalhadores, a Experiência da Zona Norte: Uma Alternativa em Saúde Pública. São Paulo: Ed.Hucitec.

DIAS, E. C., 1994. A Atenção à Saúde dos Trabalhadores no Setor Saúde (SUS), no Brasil: Realidade, Fantasia ou Utopia? Tese de Doutorado, Campinas: Faculdade de Ciências Médicas, Universidade Estadual de Campinas.

ECHTERNACHT, E., 1991. Com Quantos Paus se Faz uma Canoa ou com Quantas Mortes se Faz um Navio (Sobre o Modo de Adoecer e Morrer dos Trabalhadores Jatistas em Estaleiros do Rio de Janeiro). Dissertação de Mestrado, Rio de Janeiro: Coordenação de Pós-Graduação e Pesquisa em Engenharia, Universidade Federal do Rio de Janeiro.

FACCHINI, L. A., 1986. Proceso de Trabajo, Cambio Tecnologico y Desgaste Obrero. El Caso del Ingenio de Aucar “Adolfo Lopez Mateos". Dissertação de Mestrado, México DF.: Universidad Autonoma Metropolitana, Faculdade de Medicina.

FACCHINI, L. A., 1993. Uma contribuição da Epidemiologia: O modelo da Determinação Social 
aplicado à Saúde do Trabalhador. In: Isto é Trabalho de Gente? Vida, Doença e Trabalho no Brasil. (L.E. Rocha; J.T.P. Buschinelli \& R. Rigotto, orgs.), pp. 178-186. São Paulo: Ed.Vozes.

FERREIRA, H. P., 1994. O Perigo Silencioso, Um Estudo da Intoxicação de Trabalhadores por Vapor de Mercúrio em uma Indústria de Cloro/Soda. Rio de Janeiro. Dissertação de Mestrado, Rio de Janeiro: Escola Nacional de Saúde Pública, Fundação Oswaldo Cruz.

FROINES, J.; WEGMAN, D. \& EISEN, E., 1989. Hazard surveillance in occupational disease. American Journal of Public Health, 79 (suppl.):26-31.

GAWRYSZEWSKI, M., 1988. Uma proposta de ação sindical na luta pela saúde. Revista de Debates / Apoio Sindical, 2: 25-33.

HARRISON, R., 1990. Medical surveillance for workplace hepatotoxins. Occupational Medicine: State of Art Reviews, 5:515-530.

HEALTH \& SAFETY EXECUTIVE, 1990. Surveillance of People Exposed to Health Risks at Work. London: $\mathrm{HS}(\mathrm{G})$ series.

HERNBERG, S., 1994. Editorial. Scandinaviam Journal of Work Environmental Health, 20(special issue):5-7.

HOLANDA, M. A.; HOLANDA, M. A.; MARTINS, M. P. S.; FELISMINO, P. H. \& PINHEIRO, V. G. F., 1995. Silicose em cavadores de poços: história natural, epidemiologia e medidas de controle. Jornal de Pneumologia, 21:27-32.

KAUPPINEN, T. P., 1994. Assessment of exposure in occupational epidemiology. Scandinavian Journal of Work Environment and Health, 20:19-29.

KOSNETT, M. J., 1990. Medical surveillance for renal endpoints. Occupational Medicine: State of Art Reviews, 5:531-546.

LAURELL, A. C. \& NORIEGA, M., 1989. Processo de Produção e Saúde: Trabalho e Desgate Operário. São Paulo: Ed. Hucitec.

MACHADO, J. H.; PORTO, M. F. S. \& BRITO, J. C., 1989. Saúde e processamento de dados; o caso das doenças osteoarticulares. Cadernos de Engenharia de Produção, 5:39-73.

MACHADO, J. H.; BARCELLOS, C.; LIMA, L. \& PIVETTA, F., 1992. Arsenic exposure in the working environment of a zinc smelting plant; homogeneous group analysis. International Seminar Arsenic in the Environment and its Incidence on Health. Santiago de Chile.

MACHADO, J. H., 1995. Impact on workers health in shipbuilding industry. Safety and Health in Pratice Course. Estocolmo: National Institute of Working Life.

MATTE, T. D.; FINE, L.; MEINHARDT, T. J. \& BAKER E. L., 1990. Guidelines for medical surveillance in the workplace. Occupational Medicine: State of Art Reviews, 5:439-456.

MATTOS, U. A. O.; MELO, A. I. S. C.; ADISSI, F. \& MACHADO, J. M. H., 1995. Tolerance limits negotiation in the chemical industry. IEA Word Conference. Rio de Janeiro.

MINAYO, C. G., 1992. Projeto Integrado de Saúde do Trabalhador nas Indústrias Químicas no Rio de Janeiro. Rio de Janeiro: Centro de Estudos de Saúde do Trabalhador e Ecologia Humana, Fundação Oswaldo Cruz.
MELO, A. I. S. \& BARCELLOS, C., 1993. Mercury risk perception and assessement in a chloro-alcali plant. Heavy Metals in the Environment Congress. Edinburgh.

MENDES, E. V., 1994. O Distrito Sanitário. São Paulo: Ed. Hucitec

MENDES, R., 1986. Doenças respiratórias ocupacionais: I. Silicose. Jornal de Pneumologia, 12: 127-137.

ODDONE, I., 1986. O Ambiente de Trabalho: A Luta dos Trabalhadores pela Saúde. São Paulo: Ed. Hucitec.

OLIVEIRA, L. S. B., 1994. A Intervenção do Estado nos Ambientes de Trabalho: Origens, Evolução no Brasil e Análise Crítica da Prática da Delegacia Regional do Trabalho do Rio de Janeiro. Dissertação de Mestrado, Rio de Janeiro: Escola Nacional de Saúde Pública, Fundação Oswaldo Cruz.

PORTO, M. F. S., 1994. Trabalho Industrial, Saúde e Ecologia: Avaliação Qualitativa de Riscos Industriais em Dois Estudos de Caso na Indústria Química. Tese de Doutorado, Rio de Janeiro: Coordenação de Pós-Graduação e Pesquisa em Engenharia, Universidade Federal do Rio de Janeiro.

ROCHA, L. E.; BUSCHINELLI, J. T. P. \& RIGOTTO, R., 1993 Isto é Trabalho de Gente? Vida, Doença e Trabalho no Brasil. Vozes, São Paulo.

REBOUÇAS, A. J. A., 1986. Insalubridade Morte Lenta no Trabalho. São Paulo: Ed.Oboré.

SALGADO, P. \& FERNÍCULA, N., 1988. Noções Gerais de Toxicologia Ocupacional. São Paulo: Organização Panamericana de Saúde, Universidade Estadual de São Paulo.

SES-RJ (Seretaria de Estado de Saúde), 1991. Criação do Conselho Estadual de Saúde do Trabalhador, Resolução no 607 de 27 de dezembro de 1990. Diário Oficial do Estado do Rio de Janeiro, $2 \mathrm{de}$ janeiro:22.

SETIMI, M. M., 1995. Reintroduction of workers suffering from strain repetitive injuries in the job market/ pilot-project. Safety and Health in Pratice Course. Edinburg: National Institute of Working Life.

TAMBELLINI, A. T., 1984. A política oficial de desenvolvimento científico e tecnológico no Brasil na área de saúde-trabalho: discurso e prática. In: Ensino da Saúde Pública, Medicina Preventiva e Social no Brasil (P. M. Buss, org.) no 3, pp.13-40. Rio de Janeiro: Abrasco.

TAMBELlinI, A. T. et al., 1986. Política Nacional de Saúde dos Trabalhadores: Análises e Perspectivas. Rio de Janeiro: Abrasco/Fiocruz.

THACKER, S. B. \& BERKELMAN, R. L., 1988. Public health surveillance in the United Sates. Epidemiologic Review, 10:164-190.

WÜNSCH FILHO, V., 1992. Riscos Ocupacionais e Câncer de Pulmão. Tese de Doutorado, São Paulo: Faculdade de Saúde Pública, Universidade de São Paulo.

WÜNSCH FILHO, V.; SETTIMI, M.M. \& CARMO, J. C., 1992. Vigilância em saúde do trabalhador. III Congresso Brasileiro de Saúde Coletiva. Porto Alegre. 